\title{
Outflows of Low and High Ionization Gas in Nearby AGN
}

\author{
F. Müller Sánchez ${ }^{1}$, A. Prieto ${ }^{1}$, R. I. Davies ${ }^{2}$, and E. K. S. Hicks ${ }^{2}$ \\ ${ }^{1}$ Instituto de Astrofísica de Canarias, La Laguna, Spain \\ Email: fmueller@iac.es \\ ${ }^{2}$ Max-Planck-Institut für extraterrestrische Physik, Garching, Germany
}

Keywords. galaxies: active, galaxies: kinematics and dynamics, galaxies: nuclei, galaxies: Seyfert

We present results from a survey of nearby AGN using the adaptive optics assisted integralfield spectrometer SINFONI on the VLT. These data enable us to study at high angular resolution $\left(\sim 0 .{ }^{\prime \prime} 08\right)$ the distribution and kinematics of the low-ionization gas traced by the $\operatorname{Br} \gamma \lambda 2.17 \mu \mathrm{m}$ recombination line and [Si VI] $\lambda 1.96 \mu \mathrm{m},[\mathrm{Al} \mathrm{IX]} \lambda 2.04 \mu \mathrm{m}$, and [Ca VIII] $\lambda 2.32 \mu \mathrm{m}$, the most prominent coronal lines in the near-IR. Overall, our results show that the coronal lines extend to a radius similar to that of narrow $\mathrm{Br} \gamma$ emission, mimicking in most cases the narrow-line region (NLR). In addition, the kinematics of the highly ionized species, and most of the $\operatorname{Br} \gamma$ kinematics, is dominated by non-circular motions with higher dispersion along the ionization cones, indicating that material is being accelerated and outflowing from a region very close to the AGN.

Spectroscopic observations during the past several years suggest that the forbidden highionization lines, or coronal lines, are associated with outflows (Prieto et al. 2005; Müller Sánchez et al. 2006) and are probably formed in the NLR. However, both the size of the emitting region and the kinematics of the coronal lines are matters of actual debate. Moreover, in the central regions of active galaxies, the origin of narrow $\mathrm{Br} \gamma$ emission is uncertain, as it can be attributed either to young stars or the AGN. In order to investigate the physical properties of the low and high-ionization lines we have obtained SINFONI data of a sample of seven Seyfert galaxies on parsec scales and spectral resolution $R \sim 4000$.

Regarding to the $\mathrm{Br} \gamma$ kinematics, we have identified three cases: (i) velocity fields dominated by rotation, suggesting that $\operatorname{Br} \gamma$ is primarily associated with star formation, (ii) disturbed rotational patterns, and (iii) velocity fields dominated by non-circular motions. In the last two cases, the velocity maps of $\operatorname{Br} \gamma$ and the coronal lines are very similar, suggesting that the bulk of $\mathrm{Br} \gamma$ comes from the AGN. Interestingly, in all cases, there are clear regions along the ionization cones of the galaxies that have higher dispersion. This is interpreted as evidence of nuclear $\mathrm{Br} \gamma$ outflow (Müller Sánchez et al. 2010).

From the seven objects in our sample, four show coronal lines in their near-IR spectra. In all cases, the coronal lines extend to a radius similar to that of $\operatorname{Br} \gamma$ and their kinematics are dominated by non-circular motions. For the galaxies with existing radio images, we find that the collimated radio emission is spatially coincident with the coronal gas and use the jet orientation to constrain the outflow geometry. Biconical outflow modeling in which material is evacuated along the cone axis and tilted slightly out of the plane of the sky to match the amplitudes of blueshifted and redshifted velocities has been performed.

\section{References}

Müller Sánchez, F., et al. 2006, A\&A, 454, 481

Müller Sánchez, F., et al. 2010, in preparation

Prieto, M. A., Marco, O., \& Gallimore, J. 2005, MNRAS, 364, L28 